Ethanoanthracenes: Potential chemotherapeutics for Chronic Lymphocytic Leukaemia (CLL)

\author{
James P. Mc Keown, Clara E. Charleton, Keith Ferris, Sara Noorani, Niamh M. O'Boyle and Mary J. Meegan
}

School of Pharmacy and Pharmaceutical Sciences, Trinity College Dublin, Dublin 2, Ireland

E-mail:mmeegan@tcd.ie

\title{
Introduction
}

Chronic lymphocytic leukaemia (CLL) is the most commonly diagnosed leukaemia in the Western world, with an average age of diagnosis being over 65 years 1,2 Despite development of new targeted therapeutics; emerging resistance, pharmacoeconomic sustainability and side-effect intolerability remain significant barriers to patient care and potential disease curability

\section{Chemical Synthesis}
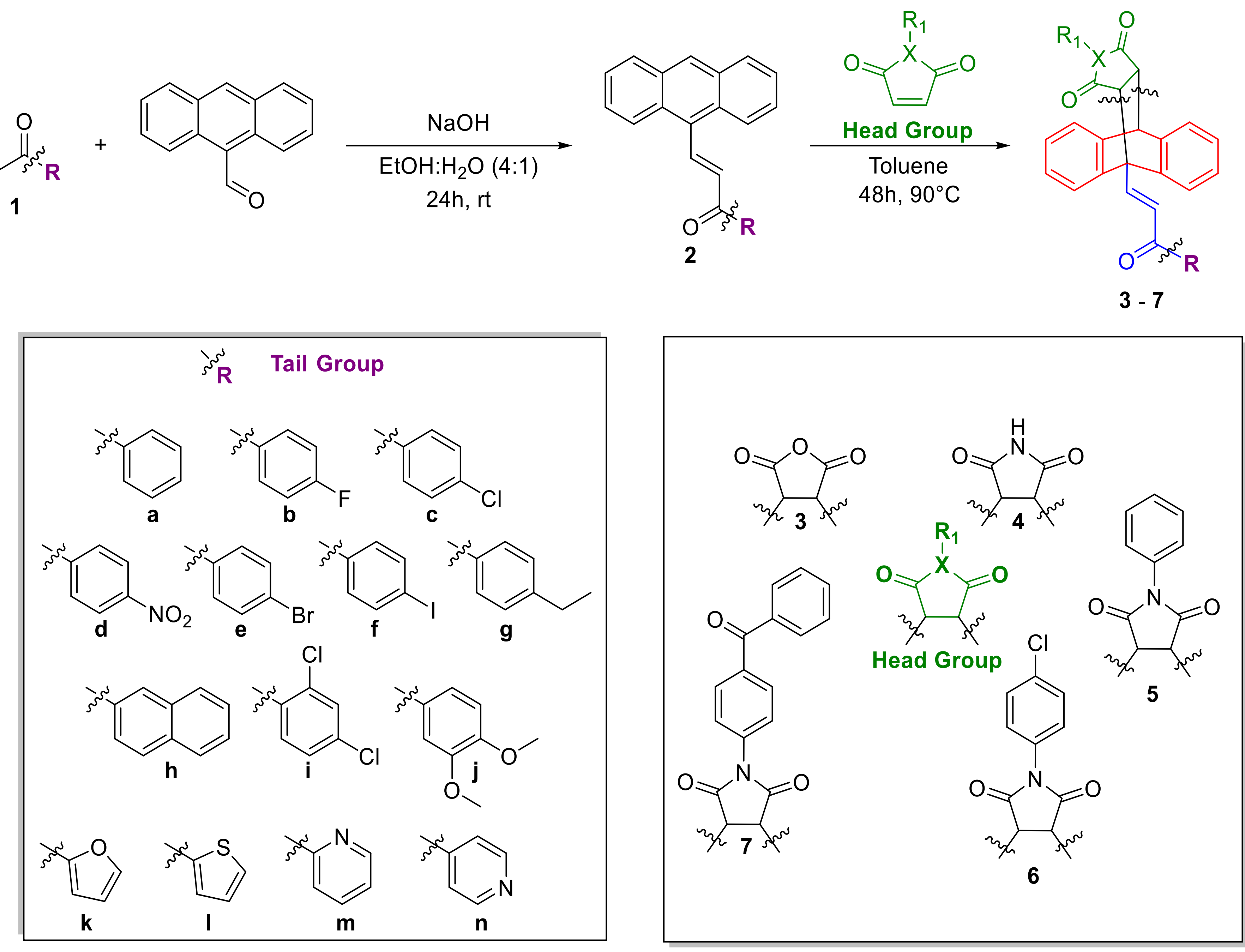

Scheme \& Figure 1: Synthesis of ethanoanthracene-based analogues with $\alpha, \beta$-unsaturated carbonyl motif (blue) via a two-step process involving Claisen Schmidt condensation followed by Diels Alder cycloaddition onto ficyclic anthracene core (red) to create ethanoanthracene derivatives. The tail groups (purple) and head groups (green) used are outlined above.

\section{Biochemical Evaluation \& SAR Elucidation}

Prior research with 2-nitrovinylanthracenes showed strong anti-proliferative proapoptototic activity in CLL lines (Fig. 2): PGA-1 (mutated Ig VH; good prognosis) and HG-3 (unmutated Ig VH; poor prognosis).
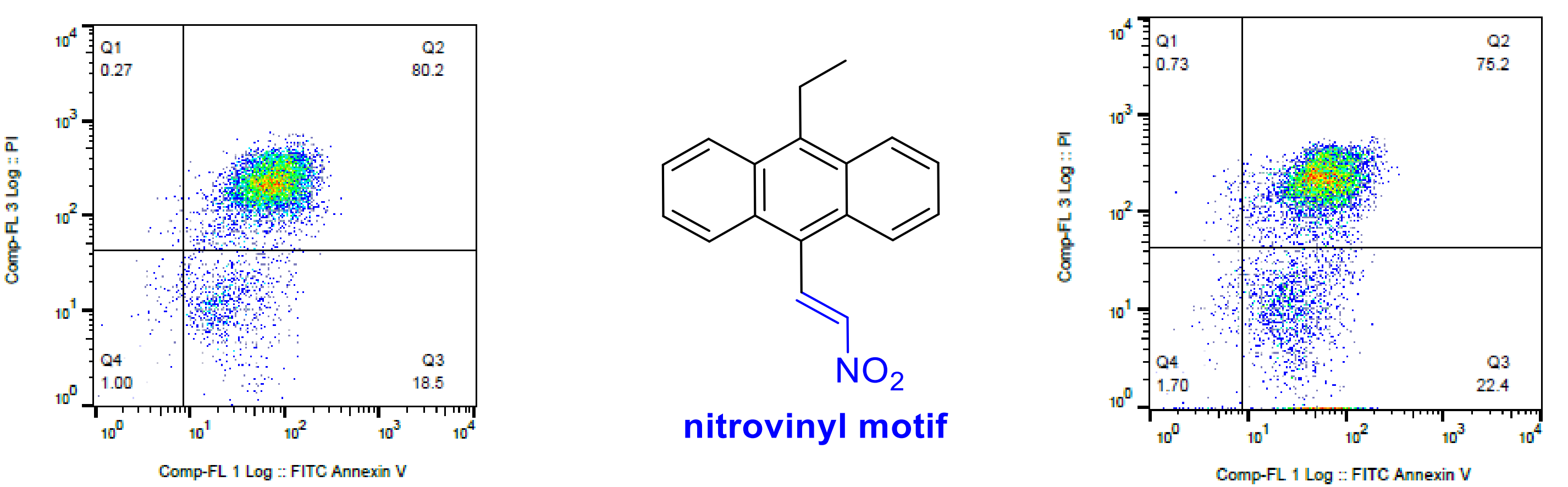

Figure 2: Annexin V/PI FACS results for HG-3 \& PGA-1 cell line treated with $10 \mu \mathrm{M}$ (E)-9-ethyl-10-(2nitrovinyll)anthracene (left \& right respectively)

Replacement of the anti-proliferative nitrovinyl with an $\alpha, \beta$-unsaturated carbonyl motif (Fig. 1) in the hope of maintaining activity, while offsetting predicted metabolic toxicity.
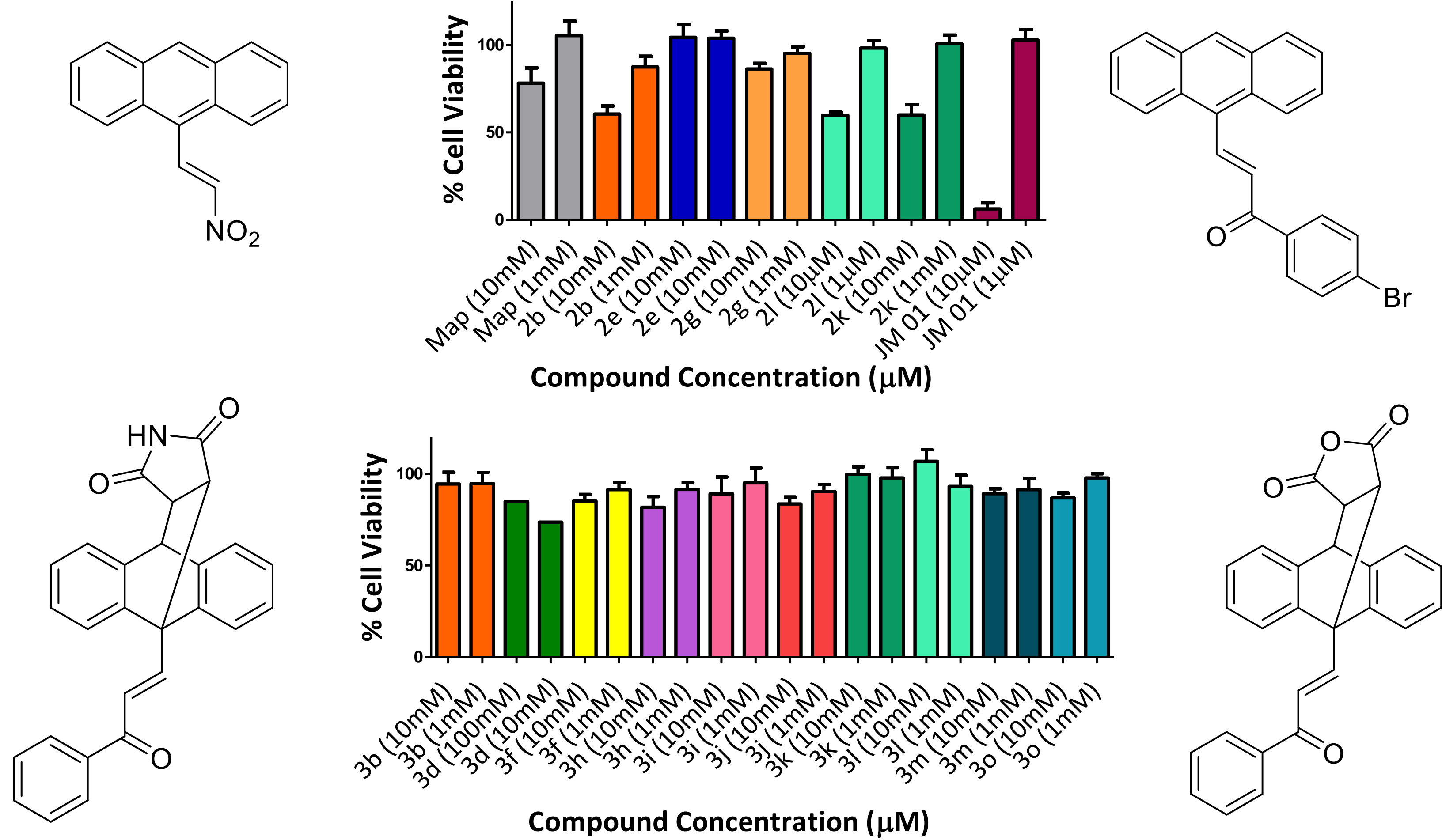

Figure 3: Alamar Blue assay at $10 \& 1 \mu \mathrm{M}$ over $24 \mathrm{~h}$ for chalcone scaffold (top) \& ethanoanthracene derivatives with maleic anhydride (bottom graph) and maleimide head groups (preceding graphs) in CLL lines: HG-3 (left) and PGA-1 (right) respectively
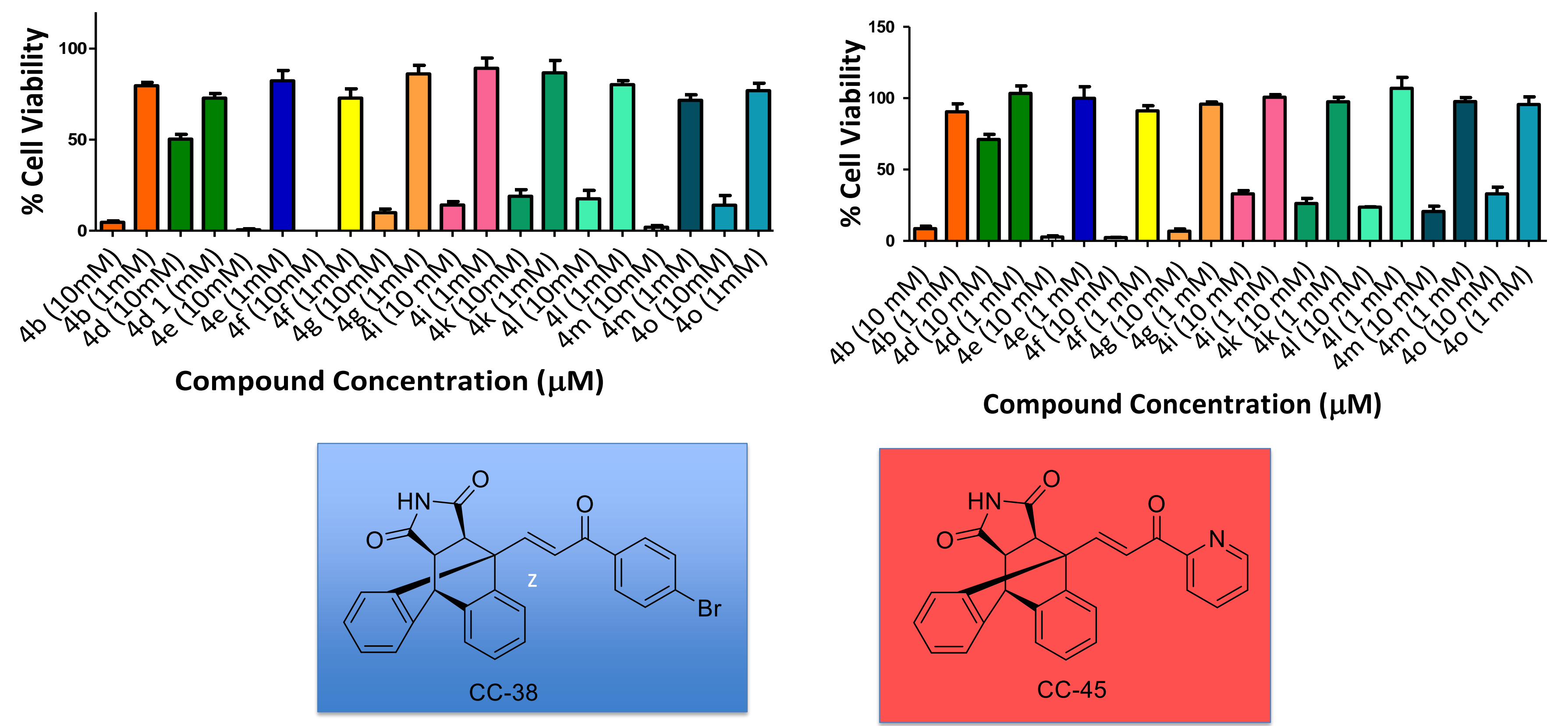

Compound Concentration ( $\mu \mathrm{M})$

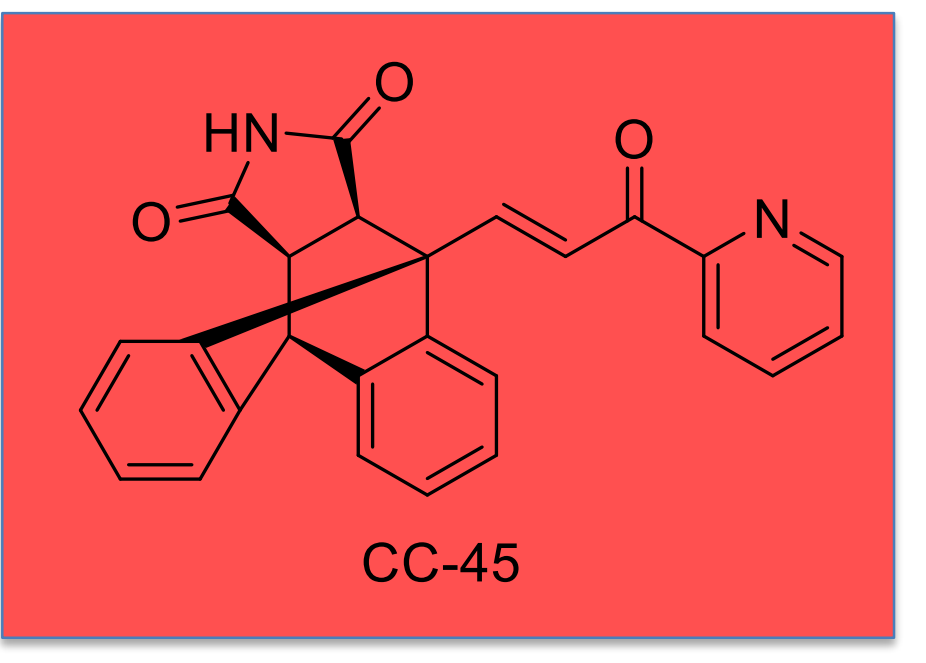

\begin{tabular}{ccc}
\hline $\begin{array}{c}\text { Compound } \\
\text { Number }\end{array}$ & $\mathrm{IC}_{50} \mathrm{HG}-3(\mu \mathrm{M})$ & $\mathrm{IC}_{50}$ PGA-1 $(\mu \mathrm{M})$ \\
\hline $\mathrm{CC}-38$ & 2.69 & 1.97 \\
\hline $\mathrm{CC}-39$ & 4.61 & 2.72 \\
\hline CC-45 & 2.39 & 1.48 \\
\hline CC-51 & 4.65 & 2.88 \\
\hline CC-52 & 4.47 & 2.77 \\
\hline Fludarabine & 28.1 & 32 \\
\hline
\end{tabular}

Figure 4 \& Table $1: \mathrm{C}_{50}$ determination of most potent ethanoanthracene chalcones compared to fludarabine ; lead compounds CC-38 and CC-45 highlighted in blue and red respectively
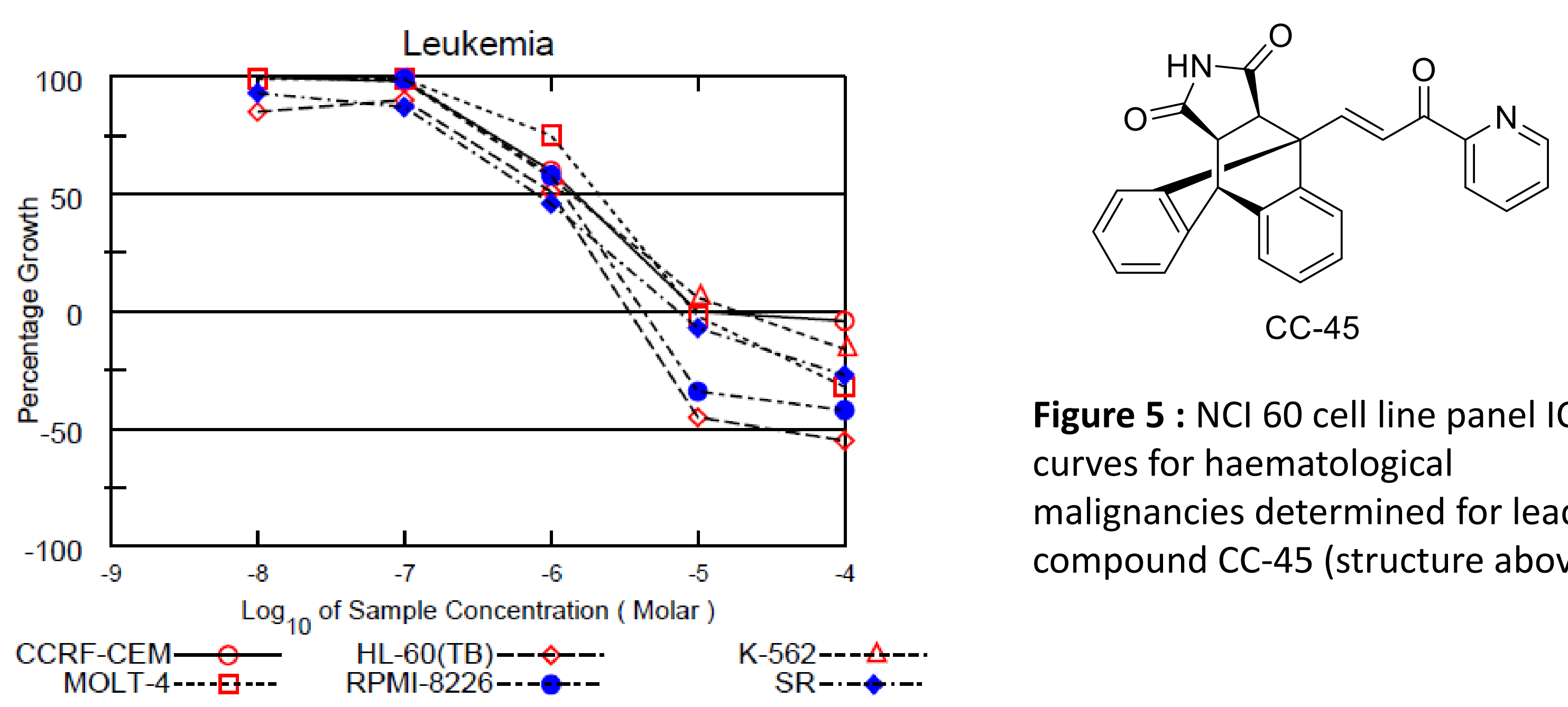

Figure 5 : NCl 60 cell line panel IC curves for haematological ermined for lead compound CC-45 (structure above)

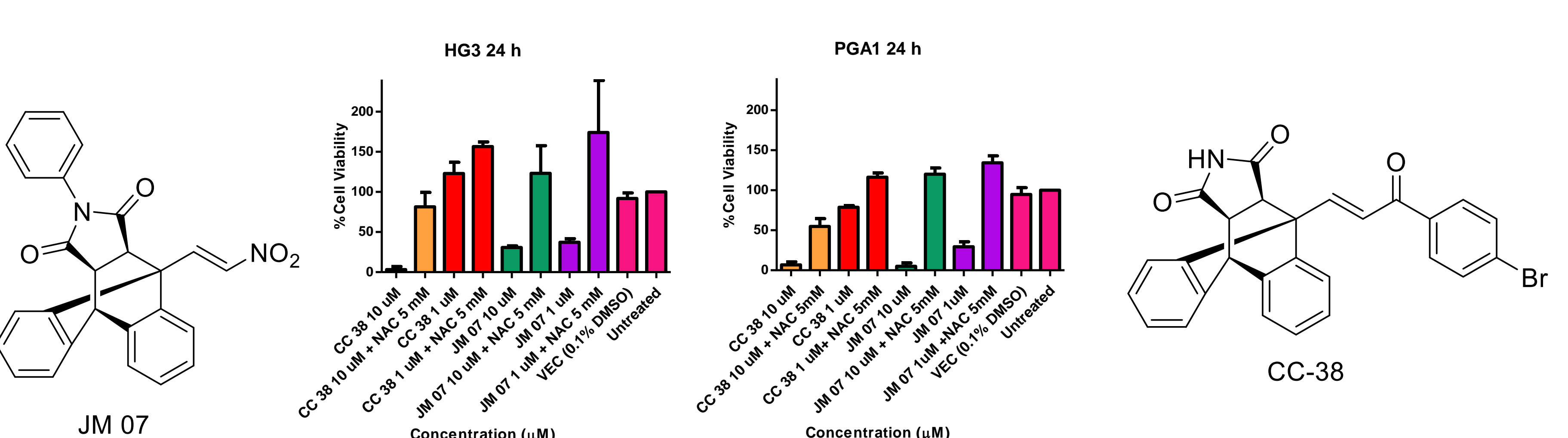

Figure 6: Reactive Oxygen Species (ROS) studies using $\mathrm{N}$-acetyl cysteine (NAC) at $5 \mathrm{mM}$ concentration across both CLL cell lines, using representative compounds of both the nitroviny and chalcone based ethanoanthracene classes (JM 07 \& CC-38 respectively)

\section{Conclusions}

$\alpha, \beta$ unsaturated ketone was observed as an effective nitrovinyl substitute for ethanoanthracene lead compounds, reaching low micromolar IC 50 values across both CLL cell lines (at least X10 as potent as fludarabine)

NCl 60 cell line COMPARE analysis of most promising compounds suggests potential effect on cellular redox mechanisms/biochemical pathways; while confirming broad anticancer activity. Haematological malignancies responded the most to treatment.

Two lead compounds JM 07 and CC-38 (representing nitrovinyl and chalcone based drug classes respectively) displayed a probable ROS dependant effect on cell viability when co-treated with antioxidants $\mathrm{N}$-acetyl cysteine or Trolox

\section{Acknowledgements}

- Trinity Postgraduate Studentship

- Staff \& Postgraduates School of Pharmacy \& School of Biochemistry (TCD)

Prof. Mc Elligott, TTMI (Trinity Translational Medicines Institute) \& School of Medicine TCD 